\title{
Developing AGVS Petri Net Control Models from Flowpath Nets
}

\author{
Suhua Hsieh and Ming-Yuan Kang, National Taiwan University, Taipei, Taiwan
}

\begin{abstract}
The paper presents a control-based Petri net (PN) modeling method that translates a high-level automated guided vehicle system (AGVS) flowpath net model into a low-level on/off control model systematically. The relation between a high-level AGVS flowpath net model and the equivalent lowlevel control net model is uniquely defined by introducing certain $1 / O$ control functions and station control macros into the net model. To ensure the uniqueness of the translation, the AGVS flowpath layout is first decomposed into constituent basic PN flowpath modules. By employing a modular concept, the wiring layout of each basic flowpath module is established. Proper $1 / O$ control functions and station control macros are introduced and incorporated into basic flowpath net modules to generate basic control net modules. The union of constituent basic control net modules systematically leads to a complete AGVS control PN model. A laboratory AGVS is used to verify the effectiveness of the proposed modeling method. The results indicate that the proposed method is capable of generating a control model directly from a given flowpath layout. Many by-products are generated by the method: wiring layout, minimum size and specification of the controller, scanning time of the control program, and so on. These features allow a designer to quickly conduct cost estimation and performance evaluation on any given flowpath layout.
\end{abstract}

Keywords: AGVS, Petri Nets, Flowpath Model, Control Model, Wiring Layout

\section{Introduction}

Petri net (PN) models have recently emerged as a popular tool for performance modeling of various discrete event systems. ${ }^{1}$ The application of the tool to model automated manufacturing systems (AMSs) is quite promising. Although PN models have been successfully applied to high-level design and analysis of AMSs, the application of these models for low-level hardware wiring and control program generation remains an area for further research. The lack of a unique relation between a high-level design layout and a low-level vehicle control logic is the major bottleneck of automating the process.

Hsieh and Shih ${ }^{2,3}$ presented an automated guided vehicle system (AGVS) design system to generate PN models for AGV system characterization.
Although the flowpath PN model constructed by Hsieh and Shih" ${ }^{2,3}$ is quite useful for AGVS design evaluation, it cannot be used for AGVS control system construction. The generation of an AGVS control program from a given flowpath layout model is a process that was conventionally done by a sequence of manual operations, including wiring layout planning, logic programming, and control ladder diagram implementation. This tedious manual process prevents a designer from quickly conducting cost estimation and performance evaluation on a given flowpath layout design. The wiring technician, who is familiar with traditional control ladder diagrams, is simply not able to define a unique logic relationship between a high-level layout design model and a low-level I/O control model. Since Petri nets are capable of modeling such a process, it is desirable to develop a procedure that can translate a high-level AGVS design model into a low-level vehicle control logic.

Researchers have addressed many interesting subjects on the design and planning of AGVS by means of analytical, simulation, and expert system models. To name a few, Takahashi and Araki ${ }^{4}$ developed programs in BASIC for AGVS simulation. Two more simulation tools, SAE/AGVS and AGVSim2, were developed by Duffau and Bloche ${ }^{5}$ and Gaskins and Tanchoco. ${ }^{6}$ Other researchers, including Gaskins, Tanchoco, and Taghaboni, ${ }^{7}$ Kaspi and Tanchoco, ${ }^{8}$ Egbelu, ${ }^{9}$ and Kim and Tanchoco, ${ }^{10}$ concentrated on developing analytical models for the flowpath optimization and/or the vehicle routes. Dhouib and $\mathrm{Kadi}^{11}$ presented a prototype expert system named KADS for collision avoidance and vehicle routing. Raju and Chetty ${ }^{12}$ and Yim and Linn ${ }^{13}$ developed Petri net based models to simulate AGVSs in FMSs. A hybrid algorithm that combines analytical, simulation, and statistical analysis is introduced by Egbelu $^{14}$ for the design of AGVSs.

A number of groups have studied manufacturing system controllers using PN models. In the early 
1980s in France, a representation method called GRAFCET was proposed as a standard specification for sequential control of manufacturing systems. This method is based on marked graphs, a special case of PNs. Hitachi in Japan developed a station controller/coordinator called SCR/SCD using an enhanced PN. ${ }^{15}$ In 1990, a stochastic PN-based representation called K-NET was developed by Kawasaki Heavy Industries, Ltd. in Japan. ${ }^{16}$ Hasegawa and Ohno, ${ }^{17}$ Bigou et al. ${ }^{18}$ Crockett et al., ${ }^{19}$ and Boucher and Jafari ${ }^{20}$ used PNs and a variation of PNs to specify shop-floor controllers. These PN-based control specifications can be implemented in different environments. It is possible to use the PN specifications as a convenient interface for sequential controllers. In this case, the PLC has a direct interaction with the physical system being controlled. The GRAFCET system exemplifies this approach. In a different scenario, one may wish to use a general-purpose computer to actually interact with the system being controlled. To do so, an interpreter is needed to generate the machine instructions directly from PN specifications. SCR/SCD is an example of this kind of environment. In the development of a flexible manufacturing system, the KNET actually goes through a few development stages, including conceptual design, preliminary design, detailed design, programming, simulation, and testing. It is a programming system that provides consistent support for all development stages.

Although research on AGVS modeling is popular, the generation of a low-level control program directly from a high-level design model is not available so far. The procedure to automate this process should include: (1) using the modular concept to decompose the flowpath layout into a basic flowpath structure and to convert each basic structure into a basic flowpath module, (2) introducing a set of wiring rules to generate basic wiring layout modules and attaching proper $\mathrm{I} / \mathrm{O}$ control functions and station control macros to each basic flowpath module to generate a basic control module, and (3) combining basic control modules to generate the complete AGVS control model and employing a lookup table to translate the AGVS control model into a corresponding set of Boolean equations and ladder diagrams. Aiming at automating this low-level control modeling process, Hsieh and Shih $^{2.3}$ and Hsieh ${ }^{21}$ proposed a system for modeling the first step that

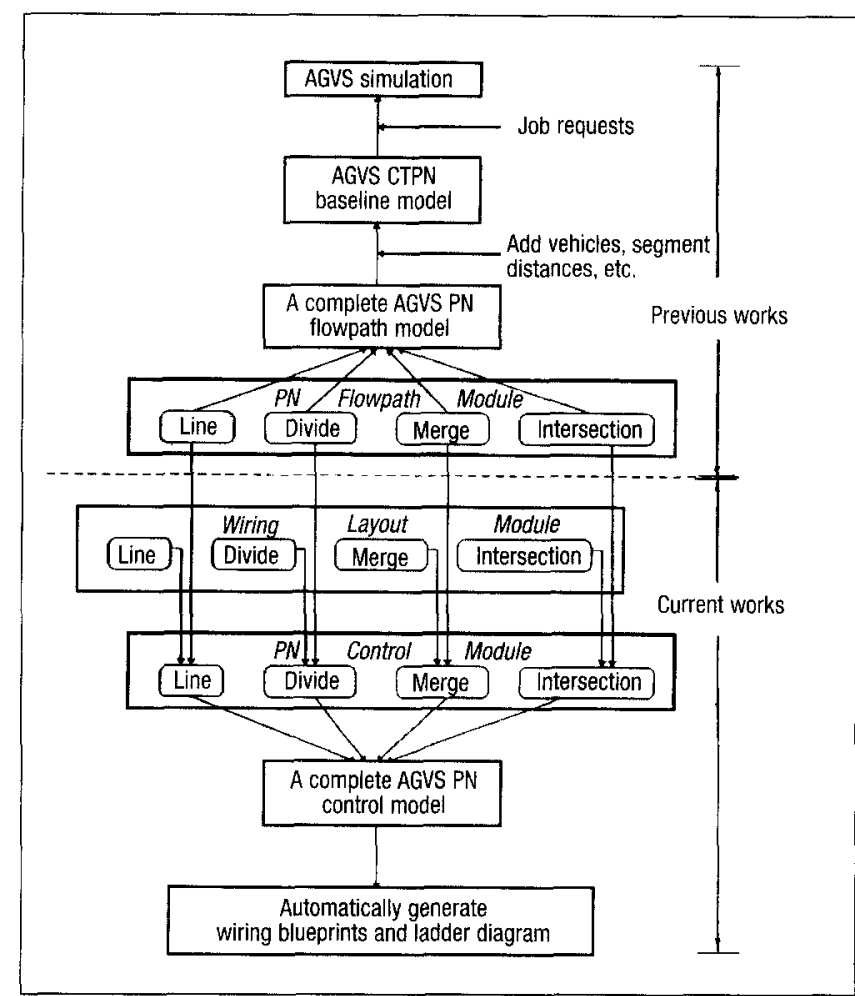

Figure 1

Modeling Scheme Diagram

generates the AGVS flowpath PN model by combining basic flowpath modules of lines, divides, merges and intersections. By coloring and timing the AGVS flowpath PN models, an AGVS colored-timed PN (CTPN) model is also generated. Based on previous work by Hsieh and Shih, ${ }^{2,3}$ this paper intends to complete the remaining modeling efforts on step (2). Figure 1 shows the proposed modeling procedure and the scope of the previous and present works.

To verify the effectiveness of the proposed theory and modeling strategy, an AGV system was designed, constructed, and used at the automation laboratory in the mechanical engineering department at National Taiwan University. Figure 2 shows the layout of the system. The system is implemented with the aid of the Mechanical Industry Research Laboratories (MIRL) of the Industrial Technology Research Institute (ITRI) in Taiwan.

\section{The AGVS and Its Hardware Controls}

An MIRL-ITRI AGV system is used for this study. ${ }^{22}$ The system adopts the single-frequency switching method for control. Functionally speaking, there are three different types of wiring loops 


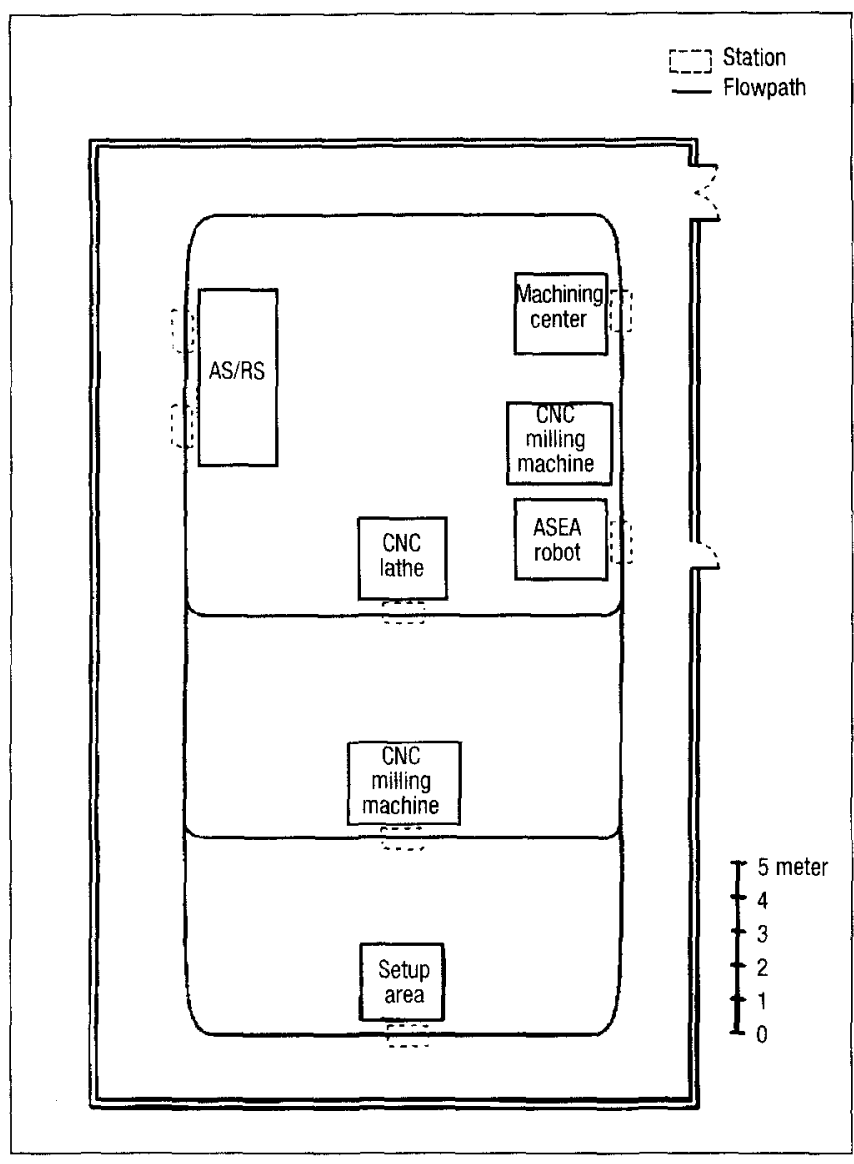

Figure 2

AGVS Layout in the Automation Laboratory at National Taiwan University

embedded on the ground in this system. The first type of wiring loop, the FL loop, works as a major guidepath line. It directs the vehicle to move based on the induced voltages. The second type of loop, the FE loop, works as an auxiliary command line that is usually embedded around the station. In general, there are two different types of FE loops, FEF and FEB. Since the FEF loop and the FEB loop cannot be both on, four different types of instructions to move vehicles are possible. These instructions are: (1) when the guidepath and the FEF loop are both on, the vehicle is instructed to move forward; (2) when the guidepath and the FEB loop are both on, the vehicle is instructed to move backward (in a unidirectional system, this instruction is not suggested to be used); (3) when the guidepath is off and the FEF loop is on, the vehicle is instructed to unload the part; and (4) when the guidepath is off and the FEB loop is on, the vehicle is instructed to load the part. The third type of wiring loop, the AW loop, works as a report line. Whenever a vehicle travels at an AW loop, a signal is generated by an equipped generator to report its current status to the controller.

For simplicity, the unidirectional wire-guided system is used for modeling this AGVS. The ladder diagram of an Allen-Bradley series 5 controller is adopted as the programming language. A zone-control method is used to prevent vehicles from collision. To reduce the number of one-step stops, a twostep-ahead forecast is used for zone control. Two different levels of control functions and commands are used by the AGVS at the laboratory. The top-level control functions are for cell-level control. A personal computer system is used as the cell controller. The vehicle activity assignments and corresponding routings are planned and issued by the cell controller and sent to the downstream programmable logic controllers (PLCs). The second-level control functions are for vehicle movement control. The PLC is used here for logic control of the hardware devices in the system. The current of the wire is switched on/off to control the vehicle activities.

\section{AGVS PN Flowpath Models}

By employing a proposed modeling procedure. Hsieh and Shih ${ }^{2.3}$ established a complete AGVS flowpath model by assembling together some basic PN flowpath modules of lines, divides, merges, and intersections. To prevent vehicles from collision, traffic control functions are introduced and embedded into basic net structures. Zones are classified into three categories: physical zone, pseudo zone, and composite pseudo zone. The physical zone is the segment of a flowpath that allows the vehicle to stop one at a time. The pseudo zone is an intersecting area of two paths in which vehicles are not allowed to stay. The composite pseudo zone is an intersecting area of several paths with a function similar to that of the pseudo zone.

Each AGVS basic flowpath module is composed of a flowpath subnet and a control-loop subnet. The flowpath subnet is the basic flowpath structure. Its traffic control function is done by the control-loop subnet. The PN representation of the AGVS has been developed by taking structural robustness into consideration. To elucidate the method, a merge structure, as shown in Figure 3, is adopted for illustration. Figure 4 is the resulting complete net of the merge structure. A zone is represented by a place in 


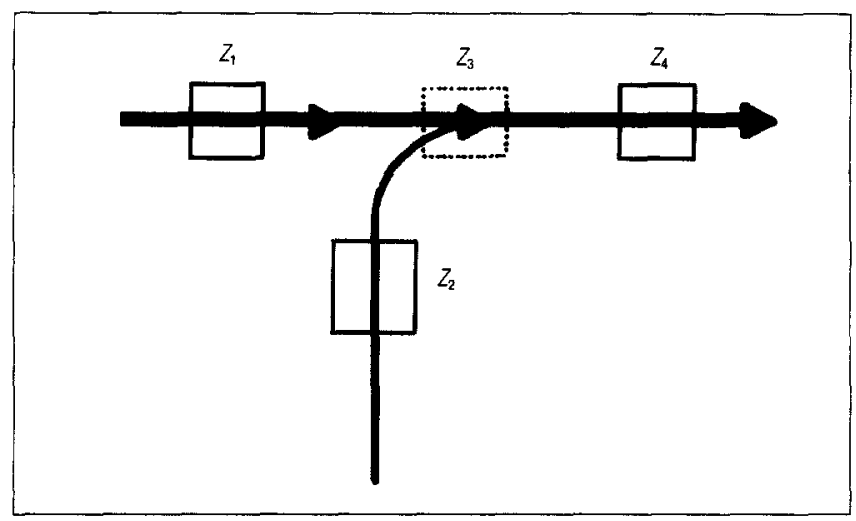

Figure 3

Flowpath Layout of a Merge Structure

the flowpath net. A vehicle is represented by a big dot token. The place in the control-loop net is used to represent the availability of a pseudo or a composite pseudo zone. The small dot token in the control place stands for the availability of the zone. The PN flowpath modules are constructed to allow the net properties of safeness, boundedness, conservation, reachability, and liveness. These Petri net properties ensure that the AGV systems being designed are collision-free, that they conserve vehicles in the system and conserve traffic control signals in each area and the whole system, and that they have path reachability and deadlock-free traffic properties.

\section{AGVS PN Control Models}

The establishment of PN control models is a twostep procedure. In the first step, the wiring layout of each basic module is established. In the second step, the basic PN control module is established. The basic PN control module is constructed by adding the signal control functions of the basic wiring layout module to the PN flowpath module. In the process of constructing a PN flowpath model, the existence of a station is not as crucial as it is in the basic wiring layout module. In other words, the existence of a station does not reflect directly on the PN flowpath model; however, it is necessary to include the wire of the station in the basic wiring layout module if there is one. A merge structure with one station allocated in zone 1, as shown in Figure 3, is used here to show the proposed modeling procedure. The wiring layout module of each basic flowpath structure, corresponding $\mathrm{PN}$ control modules, and their station control macros are obtained in sequence.

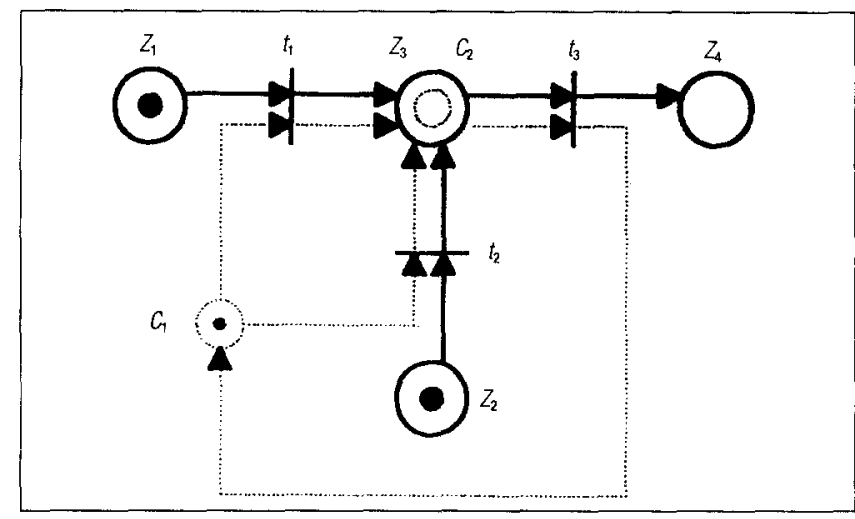

Figure 4

PN Flowpath Module of a Merge Structure

\section{Basic Wiring Layout Module}

To present basic wiring layout modules, the guidelines for implementation of basic wiring loops are addressed first. The merge structure is used to illustrate the modularized wiring principle.

\section{Implementation of the FL Loop}

The basic FL wiring loop can be designed to satisfy traffic control requirements. The main guide wire is normally on and is usually embedded in all relevant zones of the system to form major loops. When combined with secondary guide wires, the reverse magnetic flux of the secondary guide wire cancels out the flux of the main guide wire. The net effect of the combined guide wires will set the interface zone to an off state. The main guide wire is represented by a dashed line in the guide wire layout diagram. The secondary guide wire is represented by a solid line, which is usually embedded in special zone areas to turn off the current of the guidepath in the zone. The station guide wire is usually embedded near a workstation area to allow the vehicle to stop for loading or unloading. The station guide wire is also represented by a solid line in the wiring layout diagram.

Figure 5 presents FL wiring loops $L_{1}$ through $L_{6}$ for a merge structure. Loops $L_{1}$ and $L_{2}$ are two main guide wires, shown in dashed lines. Loops $L_{3}, L_{4}$, and $L_{5}$ are the secondary guide wires. Loop $L_{6}$ is the station guide wire. If zone $Z_{1}$ does not contain a station, loop $L_{6}$ shall be removed. Normally, the two main guide wires, $L_{1}$ and $L_{2}$, are on. When the guide wire $L_{3}$ is on and $L_{4}$ is off, the guidepath from $Z_{2}$ to $Z_{3}$ is on, while the guidepath from $Z_{1}$ to $Z_{3}$ is off. If $L_{5}$ is on, the guidepath from $Z_{3}$ to $Z_{4}$ is off, and no 


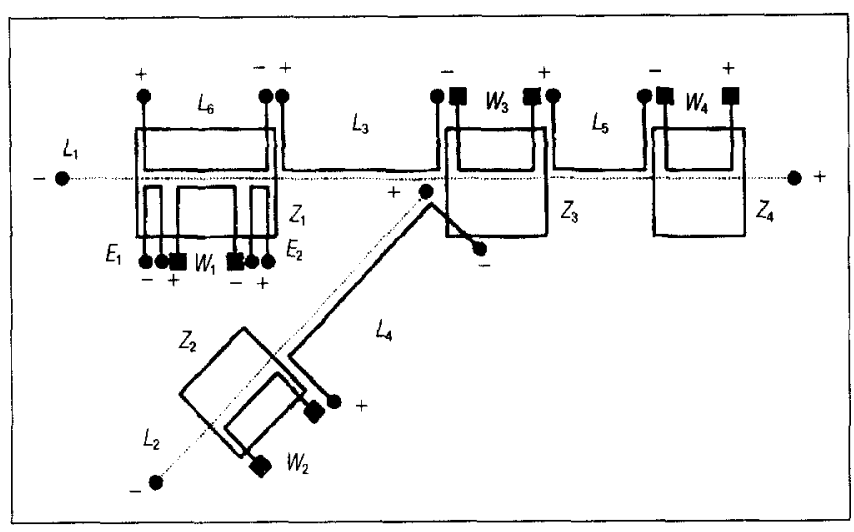

Figure 5

Wiring Diagram of a Station-Included Merge Structure

vehicle is allowed to pass through until $L_{5}$ is off. When $L_{6}$ is on, vehicles that approach $Z_{1}$ will stop at $Z_{1}$ and stay in $Z_{1}$ until $L_{6}$ is off.

\section{Implementation of FE Loop}

Based on the wiring principle of FE loops presented before, it is noted that the FE loop in a wiring layout is associated with a station and has nothing to do with the flowpath structure. Loops FEF and FEB are embedded around the station. As shown in Figure 5, zone $Z_{1}$ is a station; therefore, the FE loops are embedded around it, where $E_{1}$ is the FEF loop and $E_{2}$ is the FEB loop.

\section{Implementation of AW Loop}

As described above, when a vehicle travels to a zone where the AW loop is installed, a current in the wire is induced by the low-frequency generator onboard the vehicle. This current signals the controller immediately to accomplish the reporting task. Theoretically speaking, the AW loop has nothing to do with the flowpath structure. It may be installed wherever the traffic control is needed. For illustration, an AW loop is implemented in each zone in Figure 5 . Four different zones, $Z_{1}, Z_{2}, Z_{3}$, and $Z_{4}$, are given in the merge structure. The wiring layout of the AW loop is presented in which wires $W_{1}, W_{2}, W_{3}$, and $W_{4}$ are the report lines for zones $Z_{1}, Z_{2}, Z_{3}$, and $Z_{4}$, respectively.

\section{Wiring Layouts of Basic Modules}

It is noted so far that the wiring structure of the $\mathrm{FE}$ and $\mathrm{AW}$ loops are relatively simple compared to that of the FL loop. The structures of FE and AW are single-wired; however, the structure of the FL loop is flowpath-dependent and multiple-wired. It is constructed to meet not only the path-branching but also the modularization requirement. To simplify the wiring procedure, it is advised to follow three steps: (1) establish modularized FL wiring loops, (2) add FE loops around each station and to other appropriate locations to instruct vehicles to act properly, and (3) add the AW loop to each zone to report the status of the vehicle at the zone.

The wiring layout module for each basic flowpath structure can then be established systematically. In Figure 5, a station is located at zone 1 . When the station loop $\left(L_{6}\right)$, the FEF loop $\left(E_{1}\right)$, and the FEB loop $\left(E_{2}\right)$ are removed from Figure 5, a simple stationfree wiring layout of the merge structure is presented. Figures 6-8 show the wiring layout modules of three basic station-free flowpath structures, including the line, the divide, and the intersection, respectively.

\section{AGVS Input-Output Control Functions}

In the AGVS PN flowpath module, the vehicle movement control system and the status monitoring system are not addressed. To allow the relation between the PN flowpath module and the wiring layout module to be established, it is necessary to develop a systematic procedure to enable the functions of vehicle movement, monitor status, and control traffic. To achieve the goal, the signals of FL, FE, and AW need to be introduced into the model. It seems reasonable to treat the AW loop as an external input control signal to the controller and to treat the FL and FE loops as external output control signals to the controller.

\section{Input Control Functions}

The input control function, $I_{j}$, is usually constituted of several external input control signals, $W_{i}$ 's. As shown in Figure 5, if $W_{3}$ and $W_{4}$ are off, no vehicle

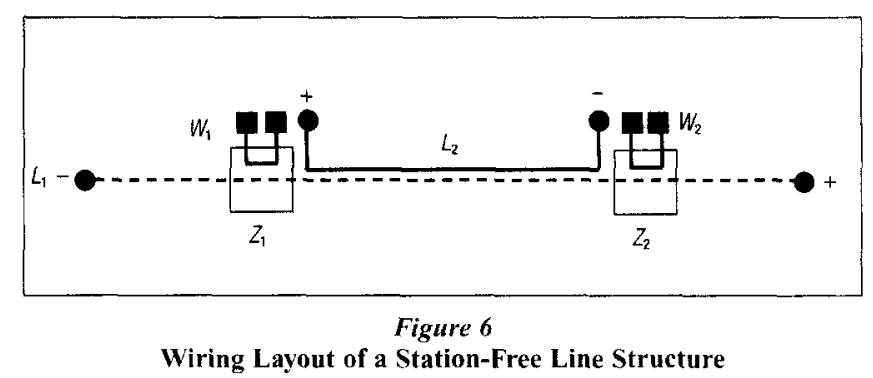




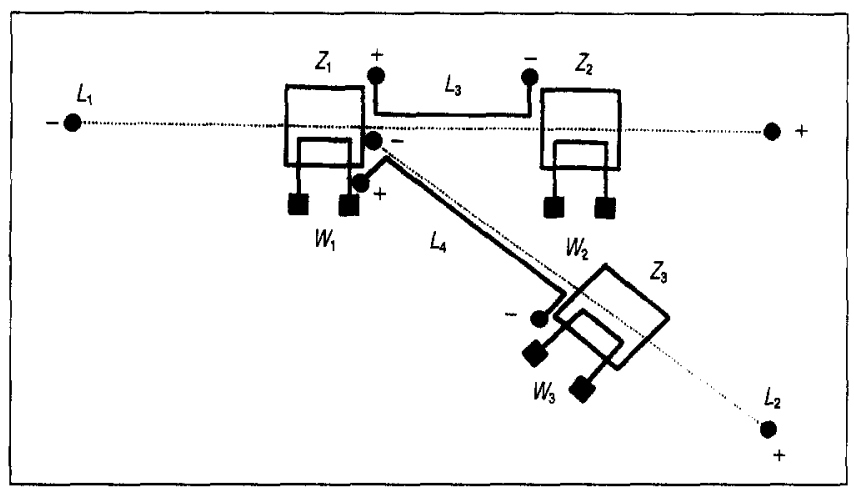

Figure 7

Wiring Layout of a Station-Free Divide Structure

is in zones $Z_{3}$ and $Z_{4}$, and the vehicle at $Z_{2}$ is safe to move. If any of $W_{3}$ and $W_{4}$ is not off, the vehicle at $Z_{2}$ is not safe to move. To enable a vehicle movement function, $I_{j}$, the values of the function-related signals, $W_{i}$ 's, need to be defined and tabulated.

\section{Output Control Commands}

The output control command from the floor-level controller is implemented by turning on/off several external output control signals. As shown in Figure 5 , when the top-level controller detects that a vehicle at $Z_{2}$ is safe to move, it issues a command to turn the $L_{3}$ on and the $L_{4}$ off. The external output control command, $O_{j}$, is composed of a set of output control signals, $L_{i}$ 's and/or $E_{i}$ 's. Whenever an output control command, $O_{j}$, is issued, a predefined set of output control signals are triggered.

\section{Station Control Macros}

When a vehicle stops at a station, it performs the task of part loading or unloading. Before the loading/unloading task is performed, the vehicle adjusts itself into the right position, which can be detected by a position sensor, $U_{1}$. Once the vehicle is in its position and ready for loading or unloading, the controller checks to see if the buffer is empty or loaded. A sensor is located in the buffer to detect the existence of the part. If the buffer is empty and the vehicle is loaded, the controller issues an unloading command to the vehicle that turns off the guidepath of the FEB loop and turns on the FEF. If the buffer is loaded and the vehicle is empty, the controller issues a loading command to the vehicle that turns off both the guidepath and the FEF loop, and turns on the FEB loop. A sensor, $U_{2}$, is used to signal the loaded state of a buffer, and a sensor, $U_{3}$, is used to

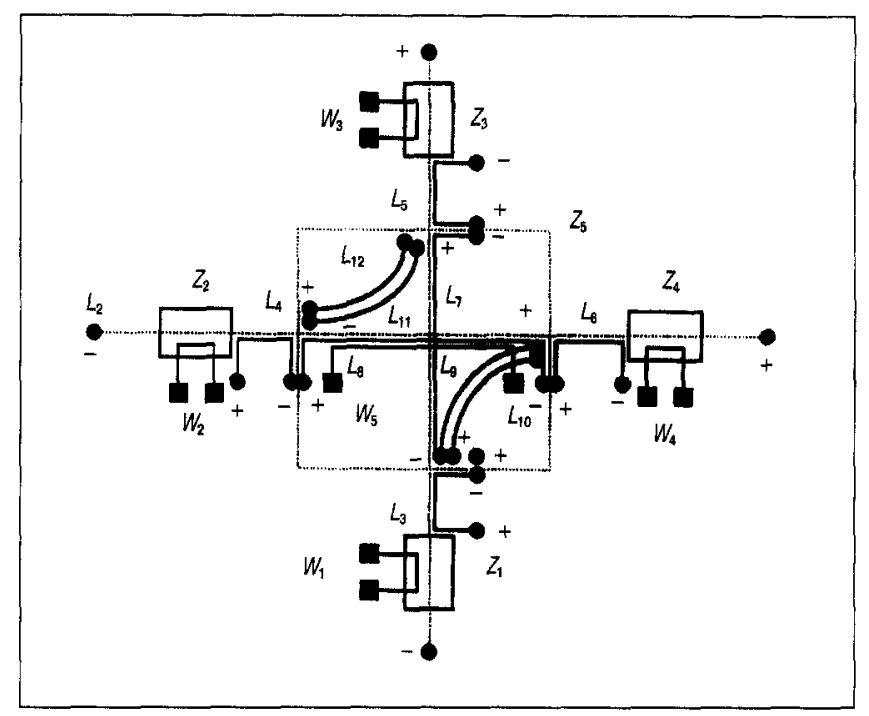

Figure 8

Wiring Layout of a Station-Free Intersection Structure

signal the empty state of a buffer. All three sensor signals, $U_{1}, U_{2}$, and $U_{3}$, are external input control signals of the station control macro to the controller. Here $R_{i j}$ and $O_{i j}$ are used to represent the input control function (constituted of $U_{1}, U_{2}$, and $U_{3}$ ) and the output control command of the station control macro, respectively.

When external input wiring control functions and output control commands, $I_{i} / R_{i j}$ and $O_{i} / O_{i j}$, are added to the transitions of a PN flowpath model, the result is a PN control model. In the control model, the transition can be fired when it is enabled and its associated external control function, $I_{i}$ or $R_{i j}$, is on. The output command, $O_{i}$ or $O_{i j}$, is directed from a transition by a directed arc with an arrow. A set of predefined external output signals are immediately issued to control the vehicle movement right after the transition is fired. These external output control signals are usually used to turn on/off the current of the FL and FE loops.

\section{Basic AGVS PN Control Module}

The basic wiring layout module and its relation to the basic PN flowpath module has been established. Now a procedure is presented for constructing PN control modules for each basic AGVS flowpath structure.

\section{Basic PN Control Module of a Merge Structure}

To employ the two-step-ahead zone control rule, one additional zone must be included into the merge structure (see Figure 4). Transition $t_{*}$ is the transition 
right behind zone $Z_{*}$, and the output place of transition $t_{r}$ is zone $Z_{4}$. It is understood that transition $t_{1}$ can be fired only when a vehicle appears in zone 1 and when zones 3 and 4 are empty. The wiring layout of the merge structure is given in Figure 5 in which the vehicle status of zones $Z_{1}, Z_{3}$, and $Z_{4}$ is reported by the wires of $W_{1}, W_{3}$, and $W_{4}$, respectively. Thus, if $W_{1}$ is on, and $W_{3}$ and $W_{4}$ are off, the transition $t_{1}$ can be fired. Since the firing of transition $t_{1}$ is related to the situation that function $W_{1}$ is on and $W_{3}$ and $W_{4}$ are off, this input function can be named $I_{1}$, defined as follows: $I_{1}$ is on when $W_{1}$ is on and $W_{3}$ and $W_{4}$ are off. $I_{1}$ is off, otherwise. By adding $I_{1}$ into the merge PN flowpath module (in Figure 4) and connecting to $t_{1}$ by a directed arc, the merge module can now recognize the status of zones. Similarly, an $I_{2}$ input function can be added to the merge module and connected to the transition $t_{2} . I_{2}$ is on whenever $W_{2}$ is on and both $W_{3}$ and $W_{4}$ are off. $I_{2}$ is off, otherwise. The external input function $I_{3}$ is enabled when $W_{3}$ is on and both $W_{4}$ and $W_{*}$ are off. Here, loop $W_{*}$ is the report line of $Z_{*}$, as mentioned before.

To enable the vehicle control, a set of output commands, $O_{1}, O_{2}$, and $O_{3}$, is defined. When output command $O_{1}$ of the merge structure is issued, loop $L_{3}$ is turned off and loop $L_{4}$ is turned on. The vehicle in zone 1 is instructed to move to zone 3. Similarly, when output command $\mathrm{O}_{2}$ is issued, loop $L_{3}$ is turned on and loop $L_{4}$ is turned off. The vehicle in zone 2 is instructed to move to zone 3 . When output command $O_{3}$ is issued, loop $L_{5}$ is turned off. The vehicle in zone 3 is instructed to move to zone 4 . The PN control module for the merge structure is presented in Figure 9. The input control functions and output control commands of the merge structure are defined and tabulated in Table 1.

By incorporating three external inputs and three external outputs into the PN flowpath merge module, the basic PN control module for a merge structure is completed. It is noted that loops $L_{6}, E_{1}$, and $E_{2}$ are station-dependent and are not presented in Table 1 . The control model does not tell how the loading and unloading commands are executed by the vehicle. The basic PN station control macro is next introduced to perform the loading and unloading commands.

\section{Basic PN Station Control Macro}

The station control macro describes the loading and unloading mechanisms at a station. When a

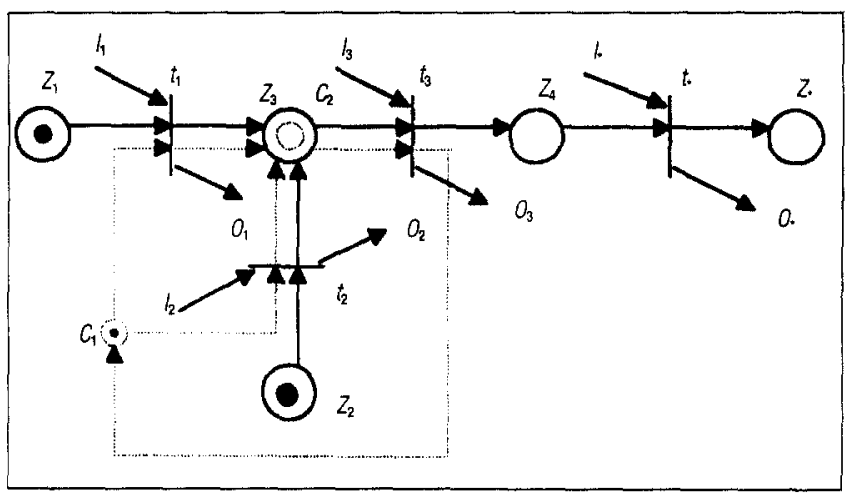

Figure 9

PN Control Module of a Merge Strueture

Table I

Definition of Each Input Control Function and Output Control Command for the Basic PN Control Model of a Merge Structure

\begin{tabular}{lll}
\hline \multicolumn{1}{c}{ Status } & On & Off \\
\hline$I_{1}$ & $W_{1}$ & $W_{3} W_{4}$ \\
$I_{2}$ & $W_{2}$ & $W_{3} W_{4}$ \\
$I_{3}$ Status & $W_{3}$ & $W_{4} W_{\star}$ \\
\hline$O_{1}$ & On function & Off \\
\hline$O_{2}$ & $L_{4}$ & $L_{3}$ \\
$O_{3}$ & $L_{3}$ & $L_{4}$ \\
\hline
\end{tabular}

Note: (1) Main guidepaths ( $L_{1}$ and $\left.L_{2}\right)$ are always "on" (2) $W_{*}$ is the report line of zone $Z_{*}$

vehicle arrives at a station, the position sensor, $U_{1}$, has to be turned on before the controller instructs the vehicle to start its unloading action. If the buffer sensor $U_{2}$ is on, the loading command is then issued by the controller to the vehicle. If the vehicle is empty, it will start its loading action. If the vehicle is not empty, it will stand by and issue an error signal to the controller. If the buffer sensor $U_{3}$ is on, the controller will issue a command to the vehicle to unload the part. If the vehicle is not empty, it will start unloading immediately. If the vehicle is empty, it will stand by and issue an error signal to the controller. The "leave" command will be issued from the controller after the vehicle has completed the loading/unloading task. The control macro of the station is developed and presented in Figure 10. The definitions of input control functions and output 
control commands are tabulated in Table 2. The definition of each place is given below.

$Z_{11}$ : vehicle has arrived at the station and is prepared to adjust its position

$Z_{12}$ : vehicle is in the right position and is prepared to load/unload the part

$Z_{13}$ : vehicle has completed the loading/unloading task and is ready to leave

$Z_{14}$ : vehicle is leaving for another zone

Figure 10 shows the detailed actions of a vehicle that approaches station $Z_{1}$. The station control macro for job loading/unloading is embedded into the basic PN control model; therefore, whenever a station macro place of the PN control model is refined, the station control macro is depicted. The relevant functions of the station are deduced and related activities of the station become apparent.

\section{Basic PN Control Modules of Other Nets}

Since the loading/unloading task is handled by the station control macro, the basic PN control module is independent of station places. The station control macro is a macro place that is usually attached to the associated zone place of the basic PN control model. By the same logic and modeling procedure, basic PN control modules of lines, divides, and intersections are established and presented in Figures 11-13, respectively. The input control functions and output control commands of each basic PN control module are defined and tabulated in Tables 3-5, respectively.

The PN control module of the intersection structure by its own nature is more complex than the other PN control modules. For example, the input

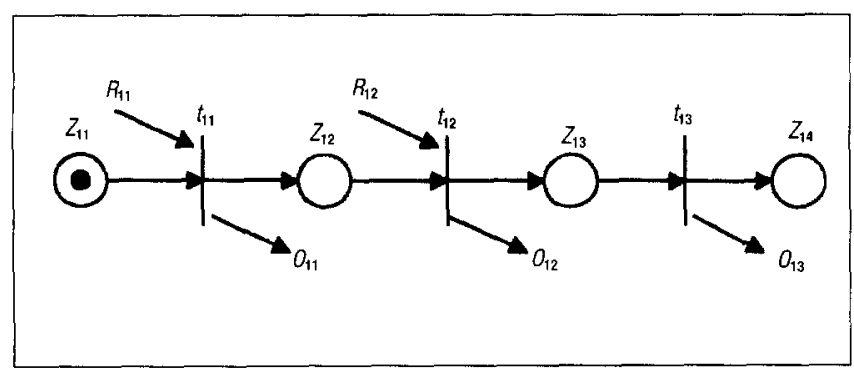

Figure 10

PN Control Station Macro
Table 2

Definition of Each Input Control Function and Output Control Command for the Basic PN Control Station Macro

\begin{tabular}{lll} 
Input function & On & Off \\
\hline
\end{tabular}
$R_{11}$

$U_{1}$

\begin{tabular}{|c|c|c|}
\hline$R_{12}$ & $U_{2}$ or $U_{3}$ & \\
\hline Output com. & On & Off \\
\hline
\end{tabular}

$O_{12}$

$$
L_{6} E_{1} \text { or } L_{6} E_{2} \quad E_{2} \text { or } E_{1}
$$

$O_{13}$

$E_{1}$ or $E_{2}$

$L_{6} E_{2}$ or $L_{6} E_{1}$

and output control functions of each zone in the intersection PN control module are obviously more tedious than those functions in other basic PN control modules. As shown in Figure 13, vehicles at $Z_{1}$ can move to either $Z_{3}$ or $Z_{4}$ through $Z_{5}$. To distinguish these two possible branches, the superscripts (1) and (2) are attached to the input control function $I_{1}$. The resulting input control functions, $I_{1}{ }^{(1)}$ and $I_{1}{ }^{(2)}$, are respectively defined as the input control function for vehicles from zone $Z_{1}$ to zone $Z_{3}$ through $Z_{5}$, and as the input control function from zone $Z_{1}$ to zone $Z_{4}$ through $Z_{5}$.

Similarly, the input control functions, $I_{2}{ }^{(1)}$ and $I_{2}^{(2)}$, are defined as the input control function for vehicles from zone $Z_{2}$ to zone $Z_{3}$ through $Z_{5}$, and as the input control function from zone $Z_{2}$ to zone $Z_{4}$ through $Z_{5}$.

The output control functions of each zone in the intersection $\mathrm{PN}$ control module are also defined by the same logic as that of the input control functions. Instead of using I's, $O$ 's are used to represent the output control functions of each of the possible branch-

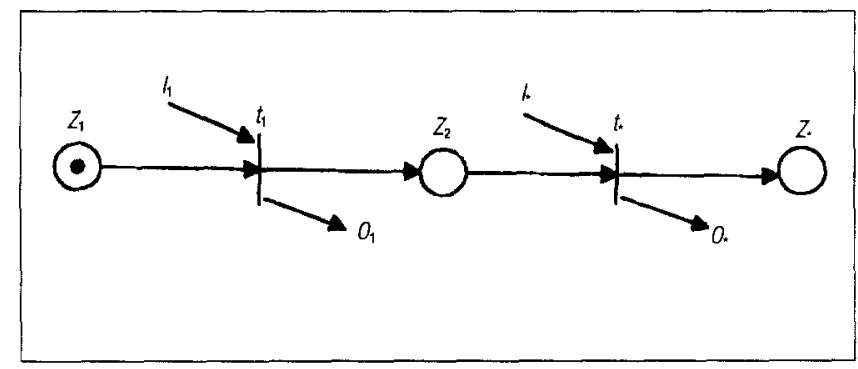

Figure 11

PN Control Module of a Line Structure 
Table 3

Definition of Each Input Control Function and Output Control Command for the Basic PN Control Model of a Line Structure

\begin{tabular}{lll}
\hline Input function & On & Off \\
\hline$I_{1}^{\text {Status }}$ Status & $W_{1}$ & $W_{2} W_{*}$ \\
\hline$O_{1}$ & On & Off \\
\hline
\end{tabular}

Note: (1) Main guidepath $\left(L_{1}\right)$ is always "on"

(2) $W_{*}$ is the report line of zone $Z$

es. The logical combinations of $I_{1}{ }^{(1)}, I_{1}{ }^{(2)}, I_{2}{ }^{(1)}, I_{2}{ }^{(2)}$, $\mathrm{O}_{1}{ }^{(1)}, \mathrm{O}_{1}{ }^{(2)}, \mathrm{O}_{2}{ }^{(1)}$, and $\mathrm{O}_{2}{ }^{(2)}$ are defined in Table 5.

\section{Model Verification}

To verify the effectiveness of the proposed control-based PN modeling method, the AGVS in the automation laboratory in the mechanical engineering department at National Taiwan University is used here as an example for illustration. The flowpath layout of the laboratory AGVS is sketched and presented in Figure 14. The layout includes 30 zone segments, two merge structures, two divide structures, and several line structures. The zones of $Z_{2}, Z_{6}$, $Z_{7}, Z_{16}, Z_{24}, Z_{26}$, and $Z_{29}$ are station zones. By assembling basic station-free wiring layout modules and connecting main guide wires, the FL and AW wiring layouts of the station-free system are obtained. By adding station guide wires and $\mathrm{FE}$ loops to $Z_{2}, Z_{6}$, $Z_{7}, Z_{16}, Z_{24}, Z_{26}$, and $Z_{29}$, a complete wiring design blueprint of the system is generated. The blueprints thus obtained are given in Figures $15 a$ and $15 \mathrm{~b}$. In Figure $15 a$, dashed lines $L_{33}$ to $L_{35}$ are main guide wires, solid lines $L_{1}$ to $L_{32}$ are secondary guide wires, and lines $L_{36}$ to $L_{42}$ are station guide wires.

The complete PN control model, as shown in

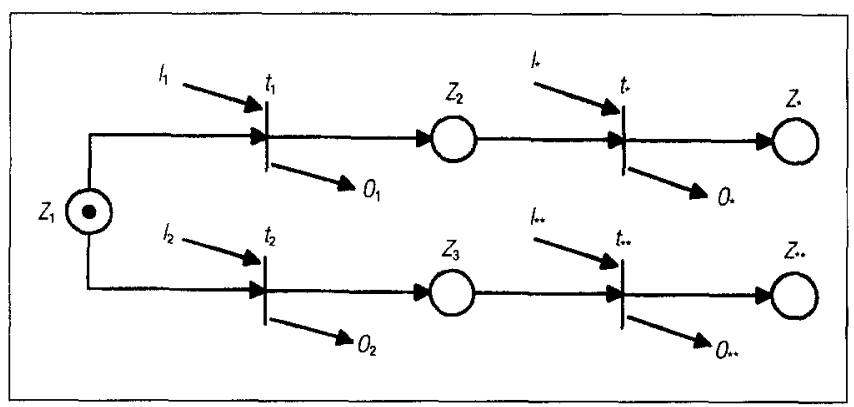

Figure 12

PN Control Module of a Divide Structure
Table 4

Definition of Each Input Control Function and Output Control

Command for the Basic PN Control Model of a Divide Structure

\begin{tabular}{ccc}
\hline Onput function & On & \multicolumn{2}{c}{ Off } \\
\hline$I_{1}$ & $W_{1}$ & $W_{2} \quad W_{*}$ \\
$W_{1}$ & $W_{3} \quad W_{* *}$ \\
\hline$O_{1}$ Status & On & Off \\
\hline$O_{2}$ & $L_{4}$ & $L_{3}$ \\
\hline
\end{tabular}

Note: (1) Main guidepaths ( $L_{1}$ and $\left.L_{2}\right)$ are always "on"

(2) $W_{*}$ and $W_{* *}$ are the report lines of $Z_{*}$ and $Z_{* *}$, respectively

Figure 16, is obtained by the combination of basic PN control modules. Since the places of $Z_{2}, Z_{6}, Z_{7}, Z_{16}$, $Z_{24}, Z_{26}$, and $Z_{29}$ are stations, if one wants to refine any of these places, the PN control station macro will need to be zoomed in. The input control functions and output control commands of the PN control model are summarized in Table 6. Total numbers of wires and sensors used in the FL, FE, and AW loops are summarized in Table 7. The results reveal the required minimum size and specification of the PLC.

The control program, which is in general a ladder diagram, can be obtained directly by translating the logic of the PN control model into a corresponding

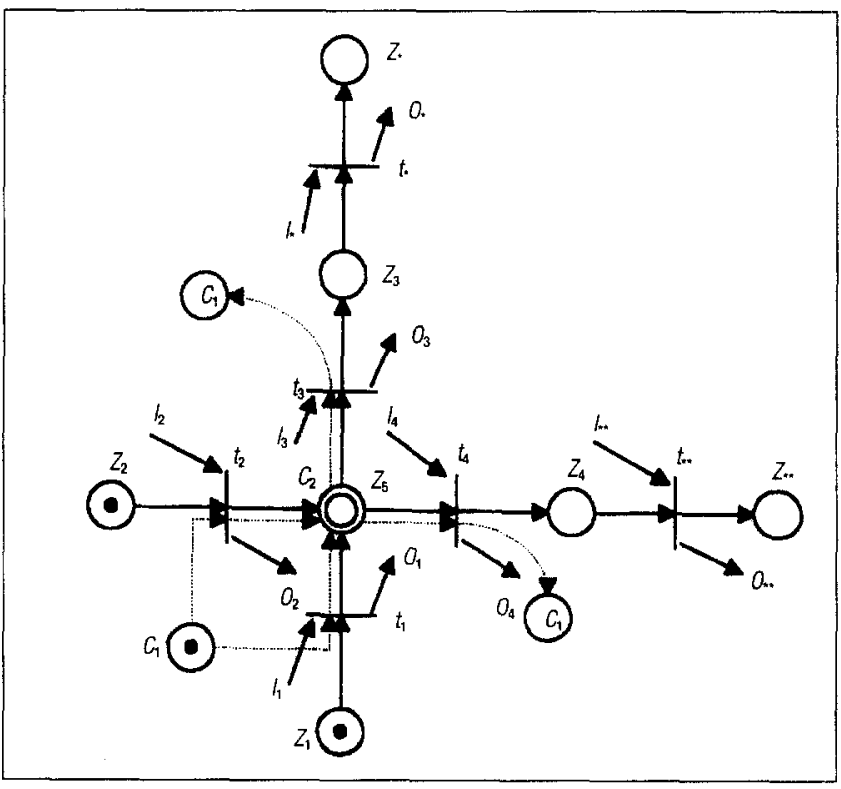

Figure 13

PN Control Module of an Intersection Structure 
Table 5

Definition of Each Input Control Function and Output Control Command for the Basic PN Control Model of an Intersection Structure

\begin{tabular}{ccc}
\hline Input function & On & Off \\
\hline$I_{1}{ }^{(1)}$ & $W_{1}$ & $W_{5} W_{3}$ \\
$I_{1}^{\left({ }^{(2)}\right.}$ & $W_{1}$ & $W_{5} W_{4}$ \\
$I_{2}{ }^{(1)}$ & $W_{2}$ & $W_{5} W_{3}$ \\
$I_{2}{ }^{(2)}$ & $W_{2}$ & $W_{5} W_{4}$ \\
$I_{3}$ & $W_{5}$ & $W_{3} W_{*}$ \\
$I_{4}$ & $W_{5}$ & $W_{4} W_{* *}$ \\
\hline Output com. & On & Off \\
\hline$O_{1}{ }^{(1)}$ & $L_{8} L_{9} L_{10}$ & $L_{3} L_{7}$ \\
$O_{1}{ }^{(2)}$ & $L_{7} L_{8} L_{9}$ & $L_{3} L_{10}$ \\
$O_{2}{ }^{(1)}$ & $L_{7} L_{8} L_{11}$ & $L_{4} L_{12}$ \\
$O_{2}{ }^{(2)}$ & $L_{7} L_{11} L_{12}$ & $L_{4} L_{8}$ \\
$O_{3}$ & & $L_{5}$ \\
$O_{4}$ & & $L_{6}$ \\
\hline
\end{tabular}

Note: (1) Main guidepaths ( $L_{1}$ and $\left.L_{2}\right)$ are always "on"

(2) $W_{*}$ and $W_{* *}$ are the report lines of $Z_{\star}$ and $Z_{* *}$, respectively

ladder format. For example, a logic "to request a vehicle at zone 2 to move forward" can be executed by firing $t_{2}$. Based on the obtained PN control model, $t_{2}$ can be fired if $I_{2}$ is true and $Z_{2}$ has a token available. This results in a situation in which $\mathrm{O}_{2}$ is true. When $\mathrm{O}_{2}$ is true, $L_{33}$ stays on (since the main guide line is initially set on) and $L_{2}$ is switched off. A corresponding set of Boolean equations can be easily obtained from the PN control model by the lookup table (Table 6) as follows:

$$
\begin{aligned}
& I_{2} \text { (on) }=W_{2} \text { (on) } * W_{2}\left(\text { off } * W_{3}\right. \text { (off) } \\
& O_{2} \text { (on) }=I_{2} \text { (on) } \\
& L_{2} \text { (off) }=O_{2} \text { (on) }
\end{aligned}
$$

It is obvious from the above equations that this process requires three control steps. By counting the number of $\mathrm{I} / \mathrm{O}$ control functions/commands and defining the system logic, one can roughly estimate

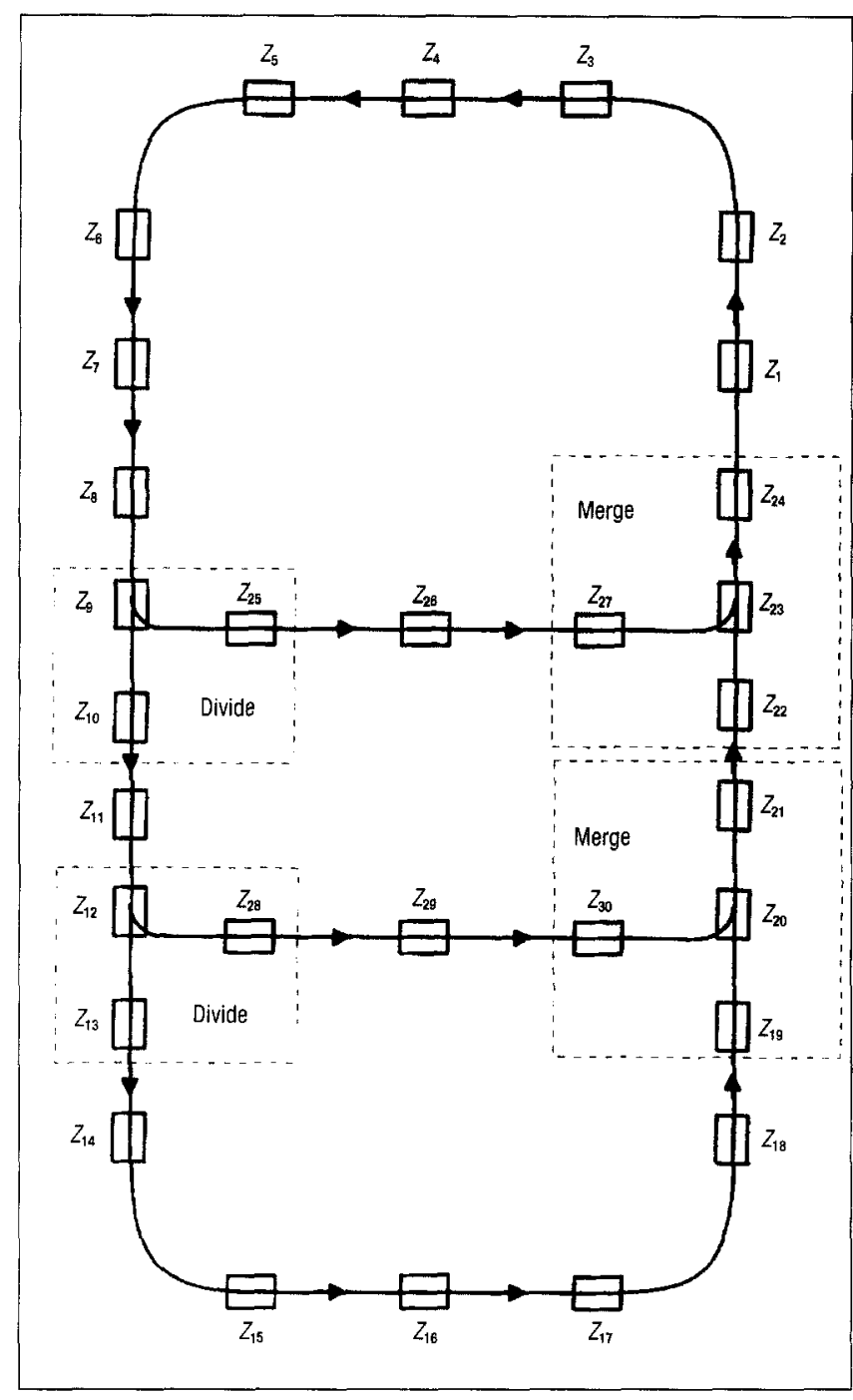

Figure 14

AGVS Flowpath Layout of the Automation Laboratory at National Taiwan University

the total required steps of a ladder diagram. In this example, the total steps required in the control program can be estimated as follows:

1. Three (3) steps are required for the logic "to turn on three main guide lines initially."

2. Three (3) or four (4) steps are required for the logic "to move a vehicle."

3. Eleven (11) steps are required for the logic "to load or unload parts."

In this application, at least 32 pairs of $\mathrm{I} / \mathrm{O}$ ports are needed for the second logic (that is, 26 pairs for 3 steps and 6 pairs for 4 steps) and 7 pairs of $\mathrm{I} / \mathrm{O}$ ports for the third logic. Therefore, a total of at least 182 


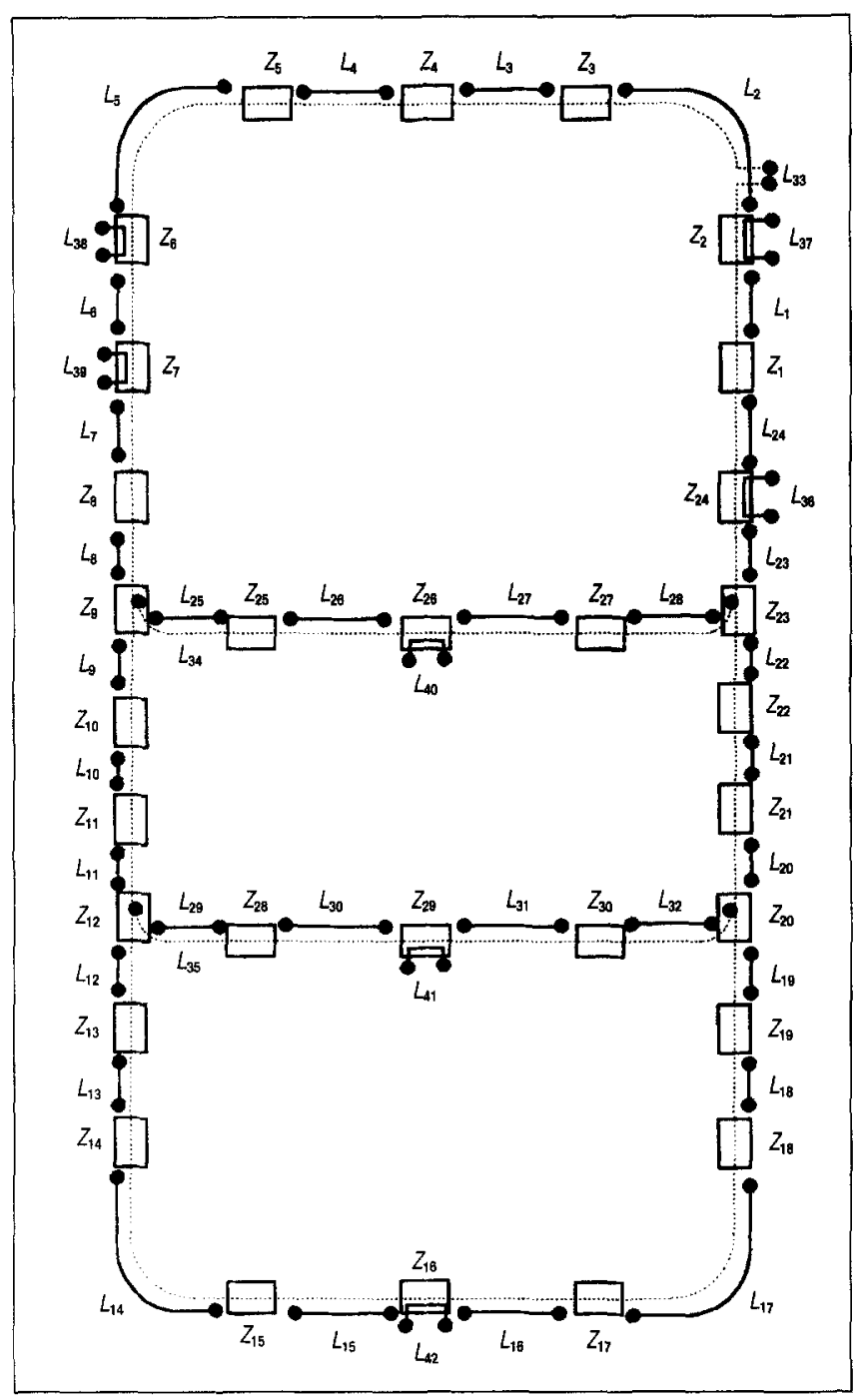

Figure 15(a)

FL Wiring Layout

steps is required in the control program. For the PLC installed in the Automation Laboratory, it takes approximately 65 microseconds on average for one control step. In other words, it will take approximately 13 milliseconds to complete a scan cycle.

The generation of an AGVS control program from a given flowpath layout is a process that was conventionally done by a sequence of manual operations, including three steps-wiring layout planning, logic programming, and ladder diagram implementation. To program the three-step process is tedious and mistake-prone and may take several months of work for technicians. The proposed PN control modeling method can make the process easily automated and mistake-free. This leads to a ready-for-control ladder diagram that can be instantly generated from

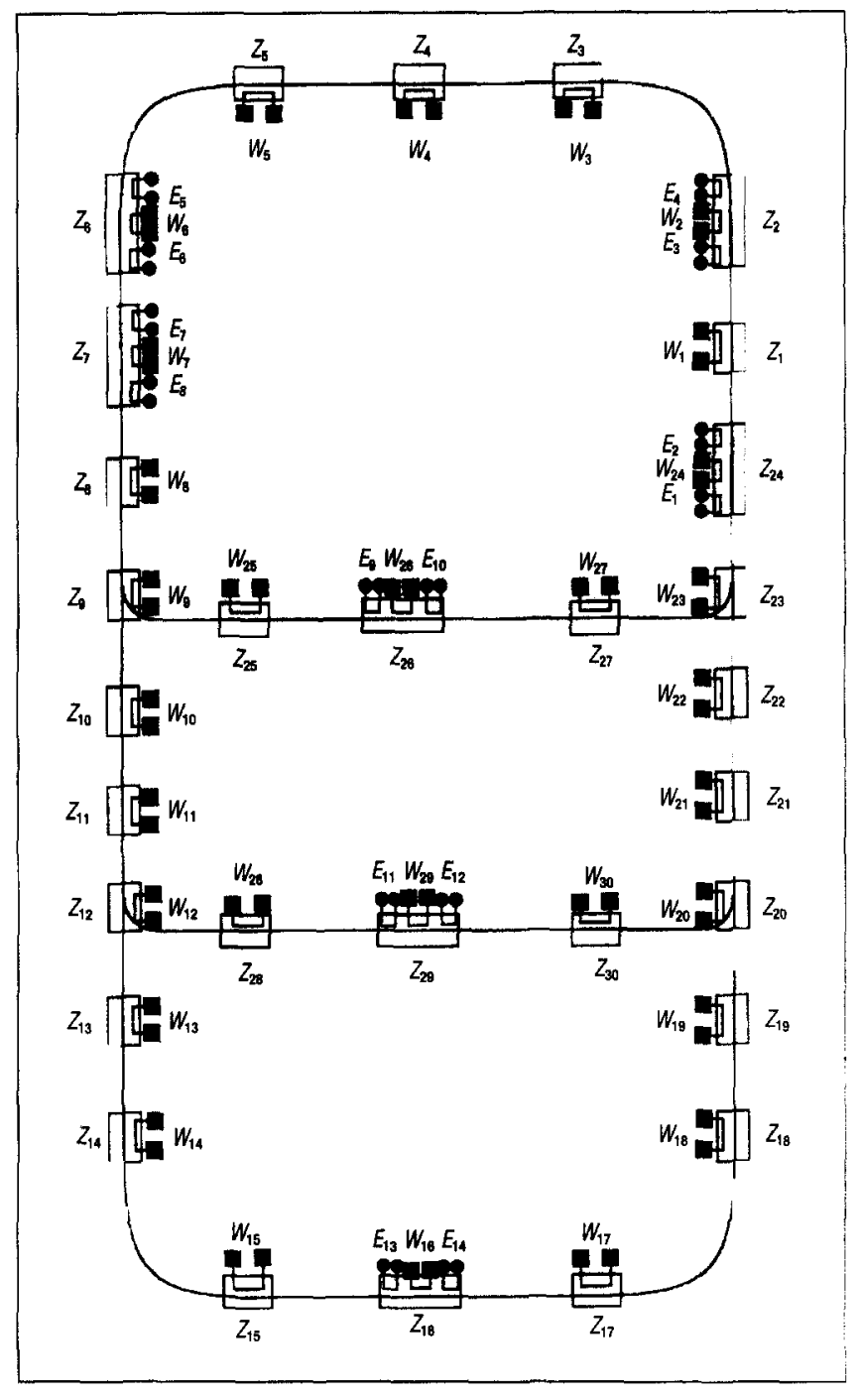

Figure 15(b)
FE and AW Wiring Layout

a given flowpath layout. A quick and reliable evaluation on a given AGVS design, including wiring layout, controller physical size (number of LO points) and performance (such as cycle time), and minimum initial investment, is then possible.

\section{Conclusion}

This paper presents a control-based Petri net modeling method that translates an AGVS flowpath layout directly into a PN control model ready for both hardware wiring implementation and software logic program control. The translation is accomplished in a two-step procedure including (1) construction of the wiring layout for each basic flowpath module and (2) construction of the basic PN control module for each basic wiring layout. A merge structure and a labora- 


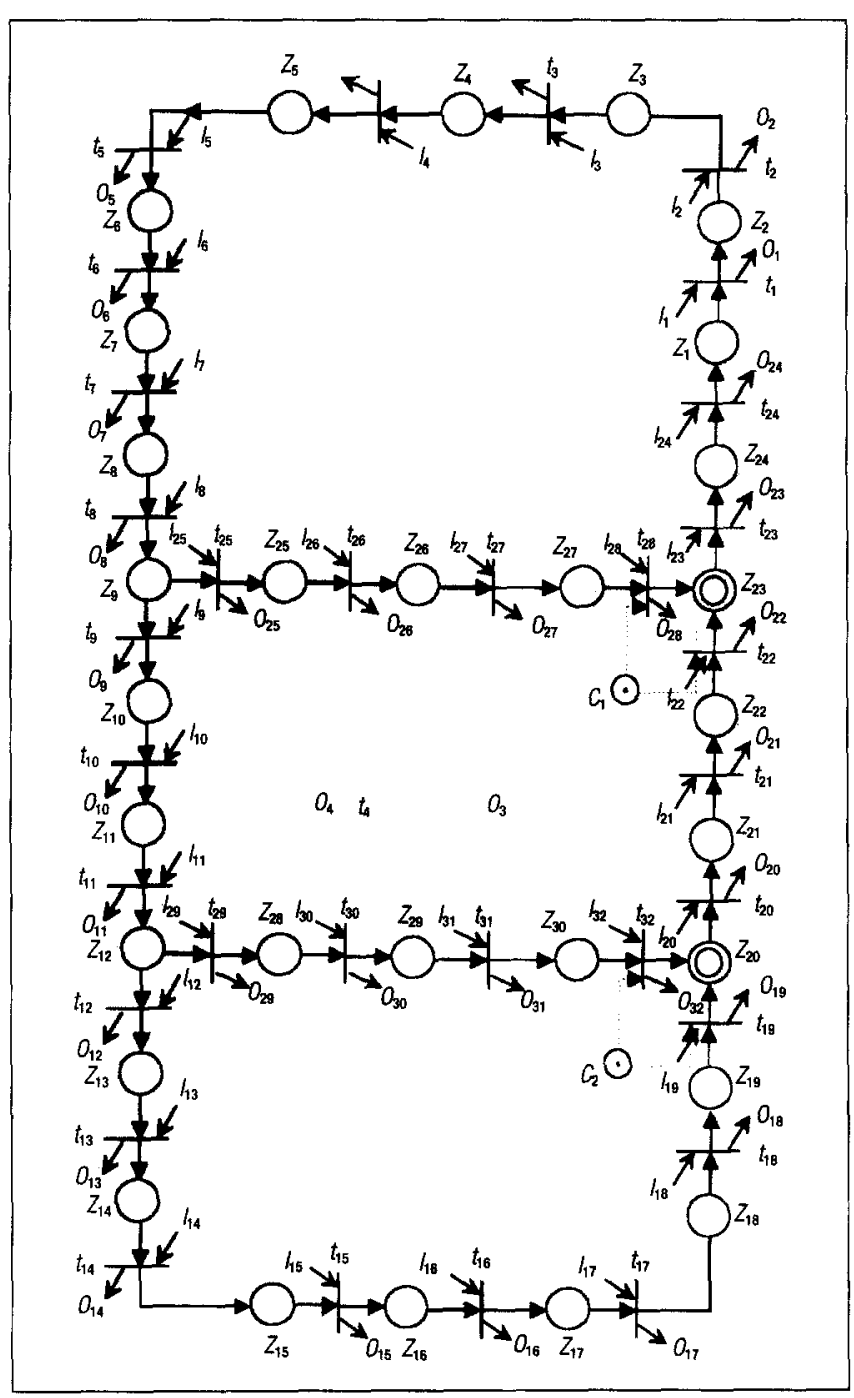

Figure 16

AGVS PN Control Model of the Automation Laboratory at National Taiwan University

tory AGVS illustrate the effectiveness of the translation process. In the course of this study, the following conclusions can be drawn:

1. For a given FL loop, there usually exist many wiring layout alternatives that can meet the design requirement. A layout technique is presented in this paper to enable the modularized modeling and implementation of wiring layouts.

2. The wiring layout of the FE loop and the AW loop are relatively simple as compared to that of the FL loop. The FE loop exists basically at stations only. The AW loop needs to be embedded in a proper zone location. When laying the wires of the FE/AW loop, only a single wiring loop is required; therefore, the FE loop and AW loop can be constructed independent of basic flowpath nets. They can be added to the FL-loop module wherever needed.

3. The wiring layout of a basic station-included traffic zone needs to be carefully designed and implemented before the PN control model is established. With a priori knowledge of a station loop in the wiring layout, the basic PN control module of a station macro can be established and attached to the associated zone place of the net.

4. Following the proposed modeling procedure, basic PN control modules can be independently constructed and assembled together to obtain the complete AGVS PN control model. The direct translation of system specifications into vehicle control logic was made possible by introducing modular concept, proper $\mathrm{I} / \mathrm{O}$ control functions and station control macros into each PN control module.

5. A quick and reliable evaluation on a given AGVS design model, including wiring layout, physical size and performance (such as cycle time) of the controller, and minimum initial investment (that is, numbers of $\mathrm{I} / \mathrm{O}$ and hardware), is now made possible by using the proposed modeling method.

\section{References}

1. M. Zhou and F. DiCesare, Petri Net Synthesis for Discrete Event Control of Manufacturing Systems (Norwell, MA: Kluwer Academic Publishers, 1993)

2. S. Hsieh and Y.J. Shih, "Automated Guided Vehicle Systems and Their Petri-Net Properties," Journal of Intelligent Mfg. (v3, 1992), pp379-390.

3. S. Hsieh and Y.J. Shih, "The Development of an AGVS Model by the Union of the Modulised Floor-Path Nets," Int 7 Journal of Advanced Mfg. Technology (v9, 1994), pp20-34.

4. T. Takahashi and T. Araki, "AGVS Design Using Computer in Factory Automation," Proc. of 3rd Int'l Conf. on Automated Guided Vehicle Systems (1985), pp 13-24

5. B. Duffau and E. Bloche, "AGVS Design in Automated Industry," Proc. of 4th Int'l Conf. on Automated Guided Vehicle Systems (1986) pp 87-102.

6. R.J. Gaskins and J.M.A. Tanchoco, "AGVSim2-A Development Tool for AGVS Controller Design," Int'l Journal of Production Research (v27, n6, 1989), pp915-926.

7. R.J. Gaskins, J.M.A. Tanchoco, and F. Taghaboni, "Virtual Flow Paths for Free-Ranging Automated Guided Vehicle Systems," Int'l Journal of Production Research (v27, n1, 1989), pp91-100.

8. M. Kaspi and J.M.A. Tanchoco, "Optimal Flow Path Design of Unidirectional AGV Systems," Int 'l Journal of Production Research (v28, n6, 1990), pp 1023-1030.

9. P. Egbelu, "Route Selection and Flow Control in a Multistage Manufacturing System with Heterogeneous Machines Within Stages," Int'l Journal of Production Research (v28, n11, 1990), pp2137-2155.

10. C.W. Kim and J.M.A. Tanchoco, "Conflict-Free Shortest-Time BiDirectional AGV Routing," Int'l Journal of Production Research (v29, 12 , 1991), pp2377-2391. 
Table 6

Definition of Each Input Control Function and

Output Control Command for the Basic PN Control

Model of the Automation Laboratory at National Taiwan University

\begin{tabular}{|c|c|c|c|c|c|c|c|c|c|c|c|}
\hline $1 / 0$ & On & Off & Status & On & Off & I/O & On & Off & Statu & On & Off \\
\hline$I_{1}$ & $W_{1}$ & $W_{2} W_{3}$ & $O_{1}$ & $L_{33}$ & $L_{1}$ & $I_{28}$ & $W_{27}$ & $W_{23} W_{24}$ & $O_{28}$ & $L_{34} L_{22}$ & $L_{28}$ \\
\hline$I_{2}$ & $W_{2}$ & $W_{3} W_{4}$ & $\mathrm{O}_{2}$ & $L_{33}$ & $L_{2}$ & $I_{29}$ & $W_{12}$ & $W_{28} W_{29}$ & $O_{29}$ & $L_{35} L_{12}$ & $L_{29}$ \\
\hline$I_{3}$ & $W_{3}$ & $W_{4} W_{5}$ & $O_{3}$ & $L_{33}$ & $L_{3}$ & $I_{30}$ & $W_{28}$ & $W_{29} W_{30}$ & $O_{30}$ & $L_{35}$ & $L_{30}$ \\
\hline$I_{4}$ & $W_{4}$ & $W_{5} W_{6}$ & $O_{4}$ & $L_{33}$ & $L_{4}$ & $I_{31}$ & $W_{29}$ & $W_{30} W_{20}$ & $O_{31}$ & $L_{35}$ & $L_{31}$ \\
\hline$I_{5}$ & $W_{s}$ & $W_{7} W_{8}$ & $O_{s}$ & $L_{33}$ & $L_{5}$ & $I_{32}$ & $W_{30}$ & $W_{20} W_{21}$ & $O_{32}$ & $L_{35} L_{19}$ & $L_{32}$ \\
\hline$I_{6}$ & $W_{6}$ & $W_{4} W_{5}$ & $O_{6}$ & $L_{33}$ & $L_{6}$ & $R_{021}$ & $U_{1}$ & & $O_{021}$ & $L_{37}$ & \\
\hline 17 & $W_{7}$ & $W_{8} w_{y}$ & $O_{7}$ & $L_{33}$ & $L_{7}$ & $R_{022}$ & $U_{2}$ or $U_{3}$ & & $O_{022}$ & $L_{37} E_{3}$ or $L_{37} E_{4}$ & $E_{4}$ or $E_{3}$ \\
\hline$I_{8}$ & $W_{8}$ & $W_{9} W_{10}$ & $O_{8}$ & $L_{33}$ & $L_{8}$ & & & & $O_{023}$ & $E_{3}$ or $E_{4}$ & $\begin{array}{c}L_{37} E_{4} \\
\text { or } L_{37} E_{3}\end{array}$ \\
\hline$I_{9}$ & $W_{9}$ & $W_{10} W_{11}$ & $O_{9}$ & $L_{33} L_{25}$ & $L_{9}$ & $R_{061}$ & $U_{4}$ & & $O_{061}$ & $L_{38}$ & \\
\hline$I_{10}$ & $W_{10}$ & $W_{11} W_{12}$ & $O_{10}$ & $L_{33}$ & $L_{10}$ & $R_{062}$ & $U_{5}$ or $U_{6}$ & & $O_{062}$ & $L_{38} E_{5}$ or $L_{38} E_{6}$ & $E_{6}$ or $E_{5}$ \\
\hline$I_{11}$ & $W_{11}$ & $W_{12} W_{13}$ & $O_{11}$ & $L_{33}$ & $L_{11}$ & & & & $O_{063}$ & $E_{5}$ or $E_{6}$ & $\begin{array}{c}L_{38} E_{6} \text { or } \\
L_{38} E_{5}\end{array}$ \\
\hline$I_{12}$ & $W_{12}$ & $W_{13} W_{1+}$ & $O_{12}$ & $L_{33} L_{29}$ & $L_{12}$ & $R_{071}$ & $U_{7}$ & & $O_{071}$ & $L_{39}$ & \\
\hline$I_{13}$ & $W_{13}$ & $W_{14} W_{15}$ & $O_{13}$ & $L_{33}$ & $L_{13}$ & $R_{072}$ & $U_{8}$ or $U_{9}$ & & $O_{072}$ & $L_{39} E_{7}$ or $L_{39} E_{8}$ & $E_{8}$ or $E_{\urcorner}$ \\
\hline$I_{14}$ & $W_{14}$ & $W_{15} W_{16}$ & $O_{14}$ & $L_{33}$ & $L_{14}$ & & & & $O_{073}$ & $E_{7}$ or $E_{8}$ & $\begin{array}{c}L_{39} E_{8} \text { or } \\
L_{39} E_{7}\end{array}$ \\
\hline$I_{15}$ & $W_{15}$ & $W_{16} W_{17}$ & $O_{15}$ & $L_{33}$ & $L_{15}$ & $R_{261}$ & $U_{10}$ & & $O_{261}$ & $L_{410}$ & \\
\hline$I_{16}$ & $W_{16}$ & $W_{17} W_{18}$ & $O_{16}$ & $L_{33}$ & $L_{16}$ & $R_{262}$ & $U_{11}$ or $U_{12}$ & & $O_{262}$ & $L_{40} E_{9}$ or $L_{40} E_{10}$ & $E_{10}$ or $E_{9}$ \\
\hline$I_{17}$ & $W_{17}$ & $W_{18} W_{19}$ & $O_{17}$ & $L_{33}$ & $L_{17}$ & & & & $O_{263}$ & $E_{9}$ or $E_{10}$ & $\begin{array}{c}L_{40} E_{10} \text { or } \\
L_{40} E_{9}\end{array}$ \\
\hline$I_{18}$ & $W_{18}$ & $W_{19} W_{20}$ & $O_{18}$ & $L_{33}$ & $L_{18}$ & $R_{\mathbf{2 9 1}}$ & $U_{13}$ & & $O_{291}$ & $L_{41}$ & \\
\hline$I_{19}$ & $W_{19}$ & $W_{20} W_{21}$ & $O_{19}$ & $L_{33}$ & $L_{19}$ & $R_{292}$ & $U_{14}$ or $U_{15}$ & & $O_{292}$ & $L_{41} E_{11}$ or $L_{41} E_{12}$ & $E_{12}$ or $E_{11}$ \\
\hline$I_{20}$ & $W_{20}$ & $W_{21} W_{22}$ & $O_{20}$ & $L_{33}$ & $L_{20}$ & & & & $O_{293}$ & $E_{11}$ or $E_{12}$ & $\begin{array}{c}L_{41} E_{12} \text { or } \\
L_{41} E_{11}\end{array}$ \\
\hline$I_{21}$ & $W_{21}$ & $W_{22} W_{23}$ & $O_{21}$ & $L_{33}$ & $L_{21}$ & $R_{161}$ & $U_{16}$ & & $O_{161}$ & $L_{42}$ & \\
\hline$I_{22}$ & $W_{22}$ & $W_{23} W_{24}$ & $O_{22}$ & $L_{33}$ & $L_{22}$ & $R_{162}$ & $U_{17}$ or $U_{18}$ & & $O_{162}$ & $L_{42} E_{13}$ or $L_{42} E_{14}$ & $E_{14}$ or $E_{13}$ \\
\hline$I_{23}$ & $W_{23}$ & $W_{2+} W_{25}$ & $O_{23}$ & $L_{33}$ & $L_{23}$ & & & & $O_{163}$ & $E_{13}$ or $E_{14}$ & $\begin{array}{c}L_{42} E_{14} \text { or } \\
L_{42} E_{13}\end{array}$ \\
\hline$I_{24}$ & $W_{24}$ & $W_{1} W_{2}$ & $O_{24}$ & $L_{33}$ & $L_{24}$ & $R_{241}$ & $U_{19}$ & & $O_{241}$ & $L_{36}$ & \\
\hline$I_{25}$ & $W_{9}$ & $W_{25} W_{26}$ & $O_{25}$ & $L_{34} L_{9}$ & $L_{25}$ & $R_{242}$ & $U_{20}$ or $U_{21}$ & & $O_{242}$ & $L_{36} E_{1}$ or $L_{36} E_{2}$ & $E_{2}$ or $E_{1}$ \\
\hline$I_{20}$ & $W_{25}$ & $W_{26} W_{27}$ & $O_{26}$ & $L_{34}$ & $L_{26}$ & & & & $O_{243}$ & $E_{1}$ or $E_{2}$ & $\begin{array}{c}L_{36} E_{2} \text { or } \\
L_{36} E_{1}\end{array}$ \\
\hline$I_{27}$ & $W_{26}$ & $W_{27} W_{23}$ & $\mathrm{O}_{27}$ & $L_{34}$ & $L_{27}$ & & & & & & \\
\hline
\end{tabular}


Table 7

Summary of Input/Output Signals of the AGVS

\begin{tabular}{cccc}
\hline $\begin{array}{c}\text { Input No. of } \\
\text { Signals }\end{array}$ & $\begin{array}{c}\text { Output } \\
\text { Input Points }\end{array}$ & $\begin{array}{c}\text { No. of Output } \\
\text { Signals }\end{array}$ & Points \\
\hline AW & 30 & FL & 42 \\
U(station) & 21 & FEB & 7 \\
Total & 51 & Total & 56 \\
\hline
\end{tabular}

11. K. Dhouib and D.A. Kadi, "Expert System for AGV Managing in BiDirectional Networks: KADS Methodology Based Approach," Int 'l Journal of Production Economics (v33, 1994), pp31-43.

12. K.R. Raju and O.V.K. Chetty, "Design and Evaluation of Automated Guided Vehicle Systems for Flexible Manufacturing Systems: An Extended Timed Petri Net-Based Approach," Int'l Journal of Production Research (v31, n5, 1993), pp1069-1096.

13. D.-S.Yim and R.J. Linn, "Push and Pull Rules for Dispatching Automated Guided Vehicles in a Flexible Manufacturing System," Int'l Journal of Production Research (v31, n1, 1993), pp43-57.

14. P. Egbelu, "Economic Design of Unit Load-Based FMSs Employing AGVs for Transport," Int'l Journal of Production Research (v31, n12, 1993), pp2753-2775.

15. N. Komoda, K. Kera, and T. Kubo, "An Autonomous, Decentralized Control System for Factory Automation," Computer (Dec. 1984), pp73-82. 16. Y. Nagao, T. Yamauchi, H. Ohta, H. Urabe, S. Matsuura, S. Kumagai, and S. Kodama, "Application of Petri Nets to FMS Programming Systems: K-NET," Proc. of IEEE International Symposium on Circuits and Systems, Singapore, June 11-14, 1991 (v2, 1991), pp922-925.
17. K. Hasegawa and H. Ohno, "On Programming of Conventional Programmable Controllers by Using Mark Flow Graph," Proc. of ISCAS 85, pp933-936.

18. J. Bigou, M. Courvoisier, H. Demmou, C. Desclaux, J. Pascal, and R. Valette, "A Methodology of Specification and Implementation of Distributed Discrete Control Systems," IEEE Trans. on Industrial Electronics IE-34 (v4, 1987), pp417-421.

19. D. Crockett, A. Desrochers, F. DiCesare, and T. Ward, "Implementation of a Petri Net Controller for a Machining Workstation," Proc. of IEEE Int'l Conf. on Robotics and Automation, Raleigh, NC, 1987. pp1861-1867.

20. T.O. Boucher and M.A. Jafari, "Design of a Factory Sequence Controller from a High Level System Specification," Journal of Mfg. Systems (v11, n6, 1993), pp401-417.

21. S. Hsieh, "Synthesis of AGVS by Colored-Timed Petri Nets," accepted for publication in Int'l Journal of Computer Integrated Mfg., 1995 (2nd rev). 22. Mechanical Industry Research Laboratory, Industrial Technology Research Inst., "AGVS Operational Manual and AGVS Operational Manual for Taiwan University Case," (Taiwan: MIRL., ITRI, 1992).

\section{Authors' Biographies}

Dr. Suhua Hsieh is an associate professor of mechanical engineering at National Taiwan University. She holds a BS in industrial engineering from National Tsing Hua University (Taiwan) and MS and PhD degrees in mechanical engineering from the University of Wisconsin-Madison. Her research and teaching interests are in factory automation, discrete event system simulation, time series analysis, and experiment design.

Ming-Yuan Kang is a $\mathrm{PhD}$ student in the mechanical engineering department at National Taiwan University, where he obtained both a BS and MS in mechanical engineering. His research interest is in the field of manufacturing systems.

\section{TWENTY-SEVENTH NORTH AMERICAN MANUFACTURING RESEARCH CONFERENCE}

\section{MAY 18-21, 1999 • UNIVERSITY OF CALIFORNIA AT BERKELEY}

Manuscript submissions are due October 1, 1998. The Scientific Committee will review all manuscripts and the Chair will notify you concerning acceptance of your paper by January 6, 1999. You will receive instructions and material on final paper preparation. Final papers are due at SME on or before February 2, 1999. All final papers must be submitted in desktop publishing format and cannot exceed six typeset pages.

Accepted papers of an archival nature will be published in the Transactions of NAMRI/SME. Accepted papers that emphasize and illustrate applications of leading-edge technology or that report work in progress, research results, or that describe a technology case study will be published in the Technical Papers of NAMRI/SME.

For more information or to receive a Paper Submission Form, contact:

Kristen Dudash

Manager, NAMRI/SME

Society of Manufacturing Engineers

One SME Drive, PO Box 930 - Dearborn, Michigan USA 48121-0930

Telephone: (313) 271-1500, ext. 532•Fax: (313) 240-8255•E-mail: dudakri@sme.org 